\title{
Self-Diffusion of Colloidal Particles in a Two-Dimensional Suspension: Are Deviations from Fick's Law Experimentally Observable?
}

\author{
Martin A. van der Hoef and Daan Frenkel \\ Foundation for Fundamental Research on Matter (FOM)-Institute for Atomic and Molecular Physics, Kruislaan 407, \\ 1098 SJ Amsterdam, The Netherlands \\ Anthony J. C. Ladd \\ Lawrence Livermore National Laboratory, Livermore, California 94550
}

(Received 26 August 1991)

\begin{abstract}
Simulations of a colloidal particle suspended in a two-dimensional fluid are reported. The dissipative and fluctuating hydrodynamic forces acting on the particle are modeled by a lattice gas. Our results indicate that large long-time tails are present in both the translational and the rotational velocity correlation functions; these are the first observations of a rotational long-time tail. The strong translational tail leads to an observable renormalization of the diffusion coefficient; our results suggest that experimental observation of the latter effect is possible.
\end{abstract}

PACS numbers: $82.70 . \mathrm{Kj}, 05.40 .+\mathrm{j}, 47.15 . \mathrm{Pn}, 51.10 .+\mathrm{y}$

Colloidal systems are interesting, both in their own right and because they can be viewed as mesoscopic models of atomic fluids. Phenomena that are difficult to observe in simple fluids can often be studied more easily in colloids. A case in point is the observation of the "longtime tail" in the velocity autocorrelation function of a tagged particle. This long-time tail was first observed in a molecular-dynamics simulation of an atomic fluid [1] and, although a quantitative theory to account for the simulation was quickly developed [2,3], direct experimental observation of the velocity autocorrelation function in an atomic fluid proved difficult, and only indirect evidence for the existence of a long-time tail could be obtained by neutron-scattering experiments [4]. By contrast, in $1973 \mathrm{Kim}$ and Matta [5] observed a long-time tail for latex spheres dispersed in air and argon, and in 1981 Paul and Pusey [6] observed a long-time tail in the diffusion of a colloidal particle by means of light-scattering experiments.

There is also good reason to try to measure the selfdiffusion of colloidal particles in an effectively two-dimensional suspension, because in two dimensions we expect that the coupling between the diffusive modes and the shear modes will change the functional form of the long-time tail, not just its amplitude as is the case in three-dimensional systems. For atomic particles the effect is extremely small and only significant from a conceptual point of view; but for colloidal particles the effect should be large and easily observable by light scattering, assuming a quasi-two-dimensional suspension can be prepared. This has not yet been accomplished, so the aim of the present paper is to demonstrate, by simulation, both the nature and the magnitude of the expected effect. It should be noted that the hydrodynamic memory effects responsible for long-time tails are absent from traditional Brownian dynamics simulations [7]. By contrast, the necessary correlations occur naturally in a lattice gas and their effects have been demonstrated by studies of tagged particle diffusion in lattice gases [8]. It was for this reason that we began developing a method for simulating colloidal suspensions [9] in which the solvent is modeled by a lattice gas; recent work [10] has shown that the dissipative hydrodynamic forces are quantitatively reproduced by this technique, even with small solid particles. In this work we have examined the diffusion of a single disk suspended in a lattice-gas fluid, to compare with the theoretical predictions of mode-coupling theory.

Long-time tails in the velocity autocorrelation function arise because the initial motion of the colloidal particle sets up a hydrodynamic flow field in the surrounding fluid, which recirculates around the particle and exerts a slowly decaying force on it. Direct evidence for this mechanism was provided by Alder and Wainwright [1] who measured the flow field around a tagged particle and observed the characteristic double-vortex structure. In order to verify that our model can reproduce vortex formation, we have given a colloidal particle suspended in the lattice gas a finite initial velocity and measured the time evolution of the flow field. The situation after 600 time steps is shown in Fig. 1; we observe that a double vortex has indeed developed. A quantitative description of long-time tails is provided by hydrodynamics [1], mode-coupling theory [2], or kinetic theory [3]. These theories predict that the velocity autocorrelation function in a two-dimensional fluid has a long-time tail of the form

$$
\left\langle v_{x}(0) v_{x}(t)\right\rangle \sim 1 /(D+v) t,
$$

where $D$ is the self-diffusion coefficient of the colloidal particle and $v$ the kinematic viscosity of the suspension. However, this expression is inconsistent, because the self-diffusion coefficient $D$ is just the time integral of the velocity autocorrelation function; if this correlation function decays as $t^{-1}$, then $D$ diverges as $\ln t$ rather than tending to a constant value as assumed in Eq. (1). As 


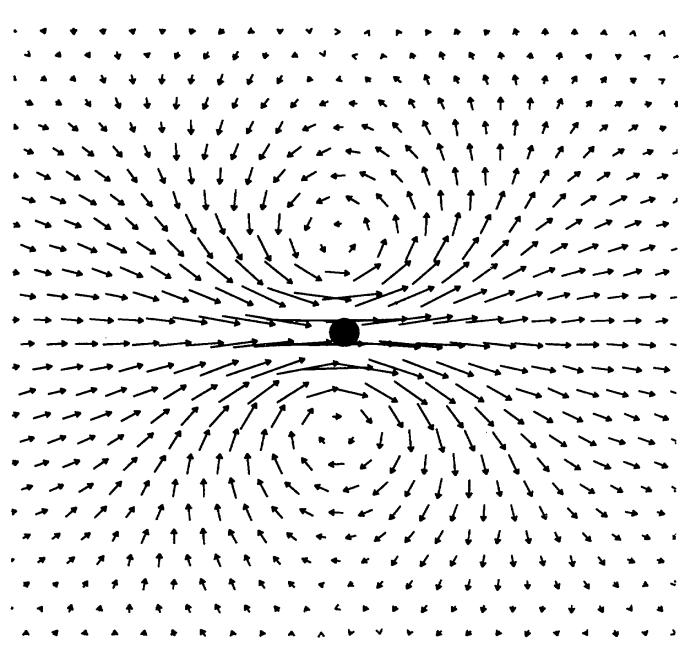

FIG. 1. Velocity field of the lattice-gas fluid, measured 600 time steps after the colloidal particle has been set in motion. The observed vortices are responsible for the "long-time tail" in the velocity autocorrelation function of the colloidal particle. The section shown measures $90 \times 90$ lattice-gas units.

was first suggested by Wainwright, Alder, and Gass [11], a self-consistent picture can be obtained by introducing time-dependent transport coefficients. In Eq. (1) Dt actually measures the mean-square displacement $\Delta(t)$ of the tagged particle. When the diffusion coefficient itself becomes time dependent, we must return to the microscopic expression for $\Delta(t)$ :

$$
\begin{aligned}
\Delta(t) & =\frac{1}{4}\left\langle[\mathbf{r}(t)-\mathbf{r}(0)]^{2}\right\rangle \\
& =\int_{0}^{t} d t^{\prime} \int_{0}^{t^{\prime}} d s\left\langle v_{x}(0) v_{x}(s)\right\rangle .
\end{aligned}
$$

The long-time tail of the velocity autocorrelation function is then given by the mode-coupling formula

$$
\left\langle v_{x}(0) v_{x}(t)\right\rangle=b /[\Delta(t)+v t],
$$

where $b=M^{*}\left\langle{ }_{v_{x}}{ }^{2}\right\rangle / 8 \pi \rho, \rho$ is the mass density of the fluid, and $M^{*}$ is the effective mass of the diffusing particle. In our simulations $v$ is independent of time, as will be seen later. Consistency of Eqs. (2) and (3) implies that at long times $\Delta(t)$ should satisfy the differential equation

$$
\frac{d^{2} \Delta(t)}{d t^{2}}=\frac{b}{\Delta(t)+v t} \text {. }
$$

The asymptotic solution of this equation, at sufficiently long times that $\Delta(t) \gg v t$, is $\Delta(t)=t \sqrt{2 b \ln t}$; the corresponding velocity autocorrelation function is proportional to $(t \sqrt{\ln t})^{-1}$. The same asymptotic form for the decay of the velocity autocorrelation function has been derived $a b$ initio by summing all the mode-coupling contributions to the long-time tail [12]. Since the long-time tail is much larger for a colloidal particle than for a tagged fluid particle, the diverging part of the denominator in Eq. (3) is also more important; consequently, the self-consistent mode-coupling effect is expected to be much more pronounced in colloidal suspensions than in tagged particle diffusion [8].

In our simulations we used a two-dimensional triangular lattice gas [13], with a parallelogram-shaped unit cell; the model differs slightly from Ref. [13] in that all possible mass- and momentum-conserving collisions are included. For convenience we work in reduced units with the mass of the lattice-gas particles, the distance between near-neighbor sites, and the time step for updating the lattice gas all equal to unity. The reduced fluid density was set to $\rho=\sqrt{12}$, corresponding to a $50 \%$ filling of the lattice. At this particular fluid density the long-time tail in the stress-stress correlation function vanishes [14]; hence the kinematic viscosity is time independent, well defined, and equal to the Boltzmann viscosity $v_{0}=0.09722$.

A moving solid body interacts with the lattice gas via additional microrules, which represent the collisions of the lattice-gas particles with the surface of the solid body [10]. On average these rules force the fluid next to the solid particle to move with the local velocity of the particle surface, modeling a hydrodynamic stick boundary condition [10]. As a consequence, the lattice-gas particles exert forces and torques on the colloidal particle which are then used to update the particle velocities and angular velocities, according to the assigned mass $M$ and moment of inertia of the solid particle. The mass of the solid particle was set to 10 times the mass of the corresponding area of fluid; in the simulations reported here the mass was of the order of 1000 . The effective mass of the colloidal particle [Eq. (4)], $M^{*}=M+2 M_{f}$, contains two contributions from the mass of the displaced fluid, $M_{f}=M / 10$; the first is the hydrodynamic-induced mass arising from the change in pressure forces as the particle accelerates, and the second comes from the mass of fluid contained by the solid particle [10] which also contributes to its inertia.

To single out the effects of colloidal particle diffusion on the long-time behavior we also performed a simulation on a system in which the colloidal particle was artificially constrained to its initial position, even though it had a nonzero velocity. We will refer to this constrained particle as "tethered," and to the diffusing particle as "free." The reason for introducing the tethered particle is that this constraint forces $\Delta(t)$ in Eq. (3) to be zero; consequently, the velocity autocorrelation function decays asymptotically as $b / v t$, the diffusion constant diverges as $(b / v) \ln t$, and there is no renormalization. This allows us to assess the quality of the simulation by comparing with a precise theoretical prediction; moreover, we can also separate the effects of vortex recirculation from the effects of the coupling between diffusive and shear modes.

The mean-square displacements $\Delta(t)$ of colloidal particles suspended in a lattice gas are plotted in Fig. 2. Results are shown for a $512 \times 512$ lattice and a colloidal par- 


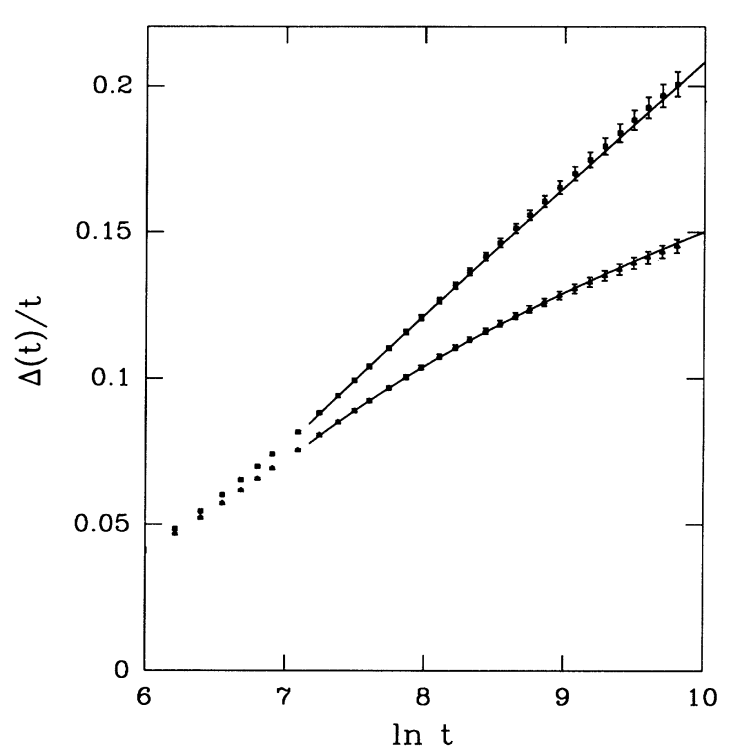

FIG. 2. Mean-square displacement divided by time vs the logarithm of the time, for a free particle (lower curve) and a tethered particle (upper curve). In the case of the tethered particle a virtual displacement, given by a time integral of the velocity, was measured. The points with error bars are the simulation data, the solid lines are the numerical solutions to Eq. (4). Note that the mean-square displacement of the free particle shows not only a reduced amplitude, but also a noticeable curvature, caused by the renormalization of the diffusion coefficient.

ticle radius of 2.5 ; they were obtained by time averaging an already equilibrated system for $50 \times 10^{6}$ time steps. Figure 2 shows that for the tethered particle, $\Delta(t) / t$ diverges logarithmically, whereas for the free particle, the pronounced curvature [in the plot of $\Delta(t) / t$ vs $\ln t$ ] indicates that there is a substantial renormalization of the mean-square displacement. Both of these effects are sufficiently large that they should be experimentally observable if a quasi-two-dimensional suspension can be prepared. The divergence of $\Delta(t) / t$ can be seen after about 1000 times steps, while self-consistent effects are quite apparent after 10000 time steps (Fig. 2). Since the diffusion coefficient is roughly 0.1 (Fig. 2) and the particle diameter is 5 lattice spacings, these times correspond to root-mean-square displacements between 4 and 10 particle diameters. Experimentally it is possible to measure single-particle mean-square displacements up to about $10^{-11} \mathrm{~m}^{2}$, so that not only the divergence of $\Delta(t) / t$, but also the self-consistent effects should be clearly. observable with $10^{-7} \mathrm{~m}$ particles. Although two-dimensional suspensions do not occur in nature, it seems likely that such a system could be approximated by dilute suspensions of colloidal particles in thin free-standing films.

Simulations with different size systems showed that the data in Fig. 2 are free of finite-size effects for up to 20000 time steps. Beyond this time, interference from

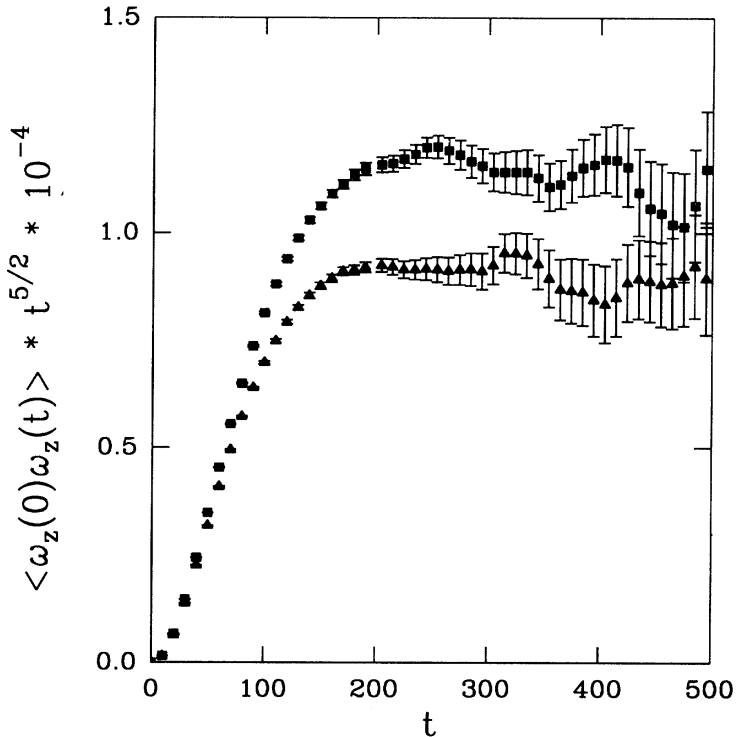

FIG. 3. Angular velocity autocorrelation function $\left\langle\omega_{z}(0)\right.$ $\left.\times \omega_{z}(t)\right\rangle$ multiplied by $t^{5 / 2}$ vs time, for the free particle (upper curve) and for the tethered particle (lower curve). The plateaus are consistent with a $t^{-5 / 2}$ decay in both cases.

periodic images causes $\Delta(t) / t$ to approach a plateau value; for very long times (200000 time steps), the diffusion coefficient of the tethered particle is consistent with a hydrodynamic calculation of the mobility of a disk in a finite-size periodic system [9]. Although the simulations cannot approach the true asymptotic region $[\Delta(t)$ $\gg v t$ ], the observed renormalization (Fig. 2) is in quantitative agreement with the self-consistent mode-coupling theory [Eq. (4)] in the intermediate-time regime where $\Delta(t) \sim v t$. However, for both the tethered and the free particle, a value of $b$ [Eq. (4)] about $8 \%$ larger than the theoretical value, $b=M^{*}\left\langle v_{x}^{2}\right\rangle / 8 \pi \rho$, was used. This discrepancy is probably due to the small size of the colloidal particle, relative to the lattice spacing. To test the effects of particle size, we repeated the simulations for a larger particle (radius 4.5); in this case we again get quantitative agreement as in Fig. 2, but now with the theoretically correct value for $b$. However, for this larger particle the vorticity takes longer to recirculate; thus the renormalization effect (for comparable times) is less pronounced than in Fig. 2.

We have also studied rotational diffusion by measuring the angular velocity autocorrelation function $\left\langle\omega_{z}(0)\right.$ $\left.\times \omega_{z}(t)\right\rangle$. We used a particle of radius 2.5 in a system of $256 \times 256$ sites and averaged over $250 \times 10^{6}$ time steps. The results suggest that there is a long-time tail in the angular velocity autocorrelation function, consistent with an asymptotic $t^{-5 / 2}$ decay (Fig. 3). Results for a particle of radius 4.5 (not shown) also suggest a $t^{-5 / 2}$ tail, although the asymptotic decay occurs at later times and is consequently more difficult to quantify. This is the first 
reported observation of a long-time tail in the angular velocity autocorrelation function, although it has been suspected theoretically for a long time. However, our results disagree with the simple hydrodynamic arguments which predict a $t^{-2}$ tail in two dimensions [15]; at the moment we have no explanation for this discrepancy. Finally, we note that Fig. 3 shows that the rotational diffusion of the free particle is faster than that of the tethered particle, suggesting that there is some coupling between translational and rotational diffusion at short to intermediate times.

We wish to acknowledge stimulating discussions with Berni Alder, Matthieu Ernst, Henk Lekkerkerker, Grzegorz Szamel, and Jan Leegwater. We thank Henk Lekkerkerker and Berni Alder for critical readings of the manuscript. This work was supported by the U.S. Department of Energy and Lawrence Livermore National Laboratory under Contract No. W-7405-Eng-48. The work of the FOM Institute is part of the scientific program of FOM and is supported by the Nederlandse Organisatie voor Wetenschappelijk Onderzoek (NWO).

[1] B. J. Alder and T. E. Wainwright, Phys. Rev. A 1, 18 (1970).
[2] M. H. Ernst, E. H. Hauge, and J. M. J. van Leeuwen, Phys. Rev. A 4, 2055 (1971).

[3] J. R. Dorfman and E. G. D. Cohen, Phys. Rev. A 6, 776 (1972).

[4] Chr. Morkel, Chr. Gronemeyer, W. Gläser, and J. Bosse, Phys. Rev. Lett. 58, 1873 (1987).

[5] Y. W. Kim and J. E. Matta, Phys. Rev. Lett. 31, 208 (1973).

[6] G. L. Paul and P. N. Pusey, J. Phys. A 14, 3301 (1981).

[7] D. L. Ermak and J. A. McCammon, J. Chem. Phys. 69, 1352 (1978).

[8] M. A. van der Hoef and D. Frenkel, Phys. Rev. Lett. 66, 1591 (1991).

[9] A. J. C. Ladd, M. E. Colvin, and D. Frenkel, Phys. Rev. Lett. 60, 975 (1988).

[10] A. J. C. Ladd and D. Frenkel, Phys. Fluids A 2, 1921 (1990).

[11] T. E. Wainwright, B. J. Alder, and D. Gass, Phys. Rev. A 4, 233 (1971).

[12] H. H.-H. Yuan and I. Oppenheim, Physica (Amsterdam) 90A, 1 (1978).

[13] U. Frisch, B. Hasslacher, and Y. Pomeau, Phys. Rev. Lett. 56, 1505 (1986).

[14] T. Naitoh, M. H. Ernst, and J. W. Dufty, Phys. Rev. A 42, 7187. (1990).

[15] N. K. Ailawadi and B. J. Berne, J. Chem. Phys. 54, 3569 (1971). 